\title{
Pruning and Quantizing Neural Belief Propagation Decoders
}

\author{
Andreas Buchberger, Student Member, IEEE, Christian Häger, Member, IEEE, \\ Henry D. Pfister, Senior Member, IEEE, Laurent Schmalen, Senior Member, IEEE, \\ and Alexandre Graell i Amat, Senior Member, IEEE
}

\begin{abstract}
We consider near maximum-likelihood (ML) decoding of short linear block codes. In particular, we propose a novel decoding approach based on neural belief propagation (NBP) decoding recently introduced by Nachmani et al. in which we allow a different parity-check matrix in each iteration of the algorithm. The key idea is to consider NBP decoding over an overcomplete parity-check matrix and use the weights of NBP as a measure of the importance of the check nodes (CNs) to decoding. The unimportant $\mathrm{CNs}$ are then pruned. In contrast to NBP, which performs decoding on a given fixed parity-check matrix, the proposed pruning-based neural belief propagation (PB-NBP) typically results in a different paritycheck matrix in each iteration. For a given complexity in terms of CN evaluations, we show that PB-NBP yields significant performance improvements with respect to NBP. We apply the proposed decoder to the decoding of a Reed-Muller code, a short low-density parity-check (LDPC) code, and a polar code. PBNBP outperforms NBP decoding over an overcomplete paritycheck matrix by $0.27-0.31 \mathrm{~dB}$ while reducing the number of required $\mathrm{CN}$ evaluations by up to $97 \%$. For the LDPC code, PB-NBP outperforms conventional belief propagation with the same number of $\mathrm{CN}$ evaluations by $0.52 \mathrm{~dB}$. We further extend the pruning concept to offset min-sum decoding and introduce a pruning-based neural offset min-sum (PB-NOMS) decoder, for which we jointly optimize the offsets and the quantization of the messages and offsets. We demonstrate performance $0.5 \mathrm{~dB}$ from ML decoding with 5-bit quantization for the Reed-Muller code.
\end{abstract}

Index Terms-Belief propagation, deep learning, min-sum decoding, neural decoders, pruning, quantization.

\section{INTRODUCTION}

$\mathbf{F}$ OR short code lengths, algebraic codes such as BoseChaudhuri-Hocquenghem $(\mathrm{BCH})$ codes and Reed-Muller (RM) codes show excellent performance under maximumlikelihood (ML) decoding. However, achieving near-ML performance using conventional methods is computationally complex. Fueled by the advances in the field of deep learning,

This work was presented in part at the IEEE International Symposium on Information Theory (ISIT) 2020.

This work was partially funded by the European Union's Horizon 2020 research and innovation programme under the Marie Skłodowska-Curie grant agreements no. 676448 and no. 749798 and by the Swedish Research Council under grant 2016-04253. Parts of the simulations were performed on resources at C3SE provided by the Swedish national infrastructure for computing.

A. Buchberger, C. Häger, and A. Graell i Amat are with the Department of Electrical Engineering, Chalmers University of Technology, Gothenburg, SE-412 96, Sweden, e-mail: \{firstname.lastname\}@ chalmers.se.

H. D. Pfister is with the Department of Electrical and Computer Engineering, Duke University, Durham, North Carolina, USA, e-mail: henry.pfister@duke.edu

L. Schmalen is with the Communications Engineering Lab, Karlsruhe Institute of Technology (KIT), 76131 Karlsruhe, Germany, e-mail: laurent.schmalen@kit.edu deep neural networks have also gained interest in the coding community [1]-[5]. In [2], belief propagation (BP) decoding is formulated in the context of deep neural networks. Instead of iterating between check nodes (CNs) and variable nodes (VNs), the messages are passed through unrolled iterations in a feed-forward fashion. Additionally, weights can be introduced at the edges, which are then optimized using stochastic gradient descent (and variants thereof). This decoding method is commonly referred to as neural belief propagation (NBP) and can be seen as a generalization of BP decoding where all individual messages are scaled by a single damping coefficient [6]. The weights in NBP can counteract the effect of short cycles by scaling messages accordingly. The concept of NBP is extended to neural offset min-sum (NOMS) in [5] by assigning an individual offset to each edge of the unrolled graph for the CN update [7], [8].

While NBP and NOMS decoding improve upon conventional BP and offset min-sum decoding, their performance is still limited by the underlying parity-check matrix. Different parity-check matrices may yield different performances. This fact has been exploited by using redundant parity-check matrices [6], [9]-[13]. In particular, [12] proposed multiple-bases belief propagation (MBBP), which selects the best decoded codeword from multiple parallel BP decoders over different parity-check matrices. For the decoding of RM codes, [13] considered applying BP with a single damping coefficient (a single weight) to the parity-check matrix composed of all minimum-weight parity checks. In [3], NBP over paritycheck matrices containing all minimum-weight parity checks was investigated. While using large, redundant parity-check matrices yields excellent performance close to ML, it suffers from high computational complexity.

In this paper, we propose pruning-based neural belief propagation (PB-NBP), a novel decoding approach based on NBP decoding to selecting the best parity-check equations for each iteration of the algorithm. The proposed approach starts with NBP decoding over the unrolled graph corresponding to a large overcomplete parity-check matrix of the linear block code. The key idea is to interpret the trained weights as a measure of the contribution of the corresponding $\mathrm{CNs}$ to the decoding process. CNs with small contribution to the decoding are then pruned. More precisely, we tie the weights of all edges emanating from a CN. CNs connected to low-magnitude-weight edges do not play an important role in the decoding process and are pruned. Pruning results in an unrolled graph with a different set of $\mathrm{CNs}$ in each $\mathrm{CN}$ layer. This corresponds to using a different 
parity-check matrix for each iteration of NBP. We investigate three variants of the PB-NBP decoder-untying all weights in the resulting (unrolled) Tanner graph, using the weights obtained during the optimization process directly, and setting all weights to one. For Reed-Muller codes, we show that PBBP decoding outperforms NBP over the overcomplete matrix and MBBP and achieves near-ML performance. Moreover, the pruning results in a lower-complexity decoder compared to NBP over the overcomplete matrix. We also give results for an LDPC code and a polar code.

We further extend the pruning concept to NOMS decoding, leading to the formulation of pruning-based neural offset minsum (PB-NOMS) decoding. For PB-NOMS we investigate the joint quantization of the weights, offsets, channel messages, and messages between layers. We use a straight-through estimator (STE) [14], [15] to define the gradient of the quantizer and let the quantization levels and thresholds be trainable. For various codes, we illustrate the performance of PB-NOMS for different quantizations.

\section{Preliminaries}

Consider a linear block code $\mathcal{C}$ of length $n$ and dimension $k$ with parity-check matrix $\boldsymbol{H}$ of size $m \times n, m \geq n-k$. If $m>n-k$, we refer to the parity-check matrix as an overcomplete matrix and denote it as $\boldsymbol{H}_{\text {oc }}$. The case $m=$ $n-k$ corresponds to a parity-check matrix with no redundant rows, which we refer to as $\boldsymbol{H}_{\text {std }}$. We denote the Tanner graph corresponding to a parity-check matrix as $\mathcal{G}=\left(\mathcal{V}_{\mathrm{v}}, \mathcal{V}_{\mathrm{c}}, \mathcal{E}\right)$, consisting of a set of $m$ CNs, $\mathcal{V}_{\mathrm{c}}=\left\{\mathrm{c}_{1}, \ldots, \mathrm{c}_{m}\right\}$, a set of $n$ VNs, $\mathcal{V}_{\mathrm{v}}=\left\{\mathrm{v}_{1}, \ldots, \mathrm{v}_{n}\right\}$, and a set of edges $\mathcal{E}$ connecting CNs with VNs.

For each $\mathrm{VN} v \in \mathcal{V}_{v}$ we define its neighborhood

$$
\mathcal{N}(\mathrm{v}) \triangleq\left\{\mathrm{c} \in \mathcal{V}_{\mathrm{c}}:(\mathrm{v}, \mathrm{c}) \in \mathcal{E}\right\}
$$

i.e., the set of all CNs connected to VN v. Equivalently, we define the neighborhood of a $\mathrm{CN} c \in \mathcal{V}_{c}$ as

$$
\mathcal{N}(\mathrm{c}) \triangleq\left\{\mathrm{v} \in \mathcal{V}_{\mathrm{v}}:(\mathrm{v}, \mathrm{c}) \in \mathcal{E}\right\} .
$$

Let $\mu_{\mathrm{v}_{i} \rightarrow \mathrm{c}_{j}}^{(\ell)}$ be the message passed from $\mathrm{VN} \mathrm{v}_{i} \in \mathcal{V}_{\mathrm{v}}$ to $\mathrm{CN}$ $\mathrm{c}_{j} \in \mathcal{V}_{\mathrm{c}}$ and $\mu_{\mathrm{c}_{j} \rightarrow \mathrm{v}_{i}}^{(\ell)}$ the message passed from $\mathrm{CN} \mathrm{c}_{j} \in \mathcal{V}_{\mathrm{c}}$ to VN $v_{i} \in \mathcal{V}_{v}$ in the $\ell$-th decoding iteration. For BP decoding, the $\mathrm{VN}$ and $\mathrm{CN}$ updates are

$$
\mu_{\mathrm{v}_{i} \rightarrow \mathrm{c}_{j}}^{(\ell)}=\mu_{\mathrm{ch}, \mathrm{v}_{i}}+\sum_{\mathrm{c} \in \mathcal{N}\left(\mathrm{v}_{i}\right) \backslash \mathrm{c}_{j}} \mu_{\mathrm{c} \rightarrow \mathrm{v}_{i}}^{(\ell)}
$$

and

$$
\mu_{c_{j} \rightarrow v_{i}}^{(\ell)}=2 \tanh ^{-1}\left(\prod_{v \in \mathcal{N}\left(c_{j}\right) \backslash v_{i}} \tanh \left(\frac{1}{2} \mu_{v \rightarrow c_{j}}^{(\ell)}\right)\right)
$$

respectively, where $\mu_{\mathrm{ch}, \mathrm{v}_{i}}$ is the channel message. For binary transmission over the additive white Gaussian noise channel

$$
\mu_{\mathrm{ch}, \mathrm{v}_{i}} \triangleq \ln \frac{p_{Y \mid B}\left(y_{i} \mid b_{i}=0\right)}{p_{Y \mid B}\left(y_{i} \mid b_{i}=1\right)}=\frac{2 y_{i}}{\sigma^{2}}
$$

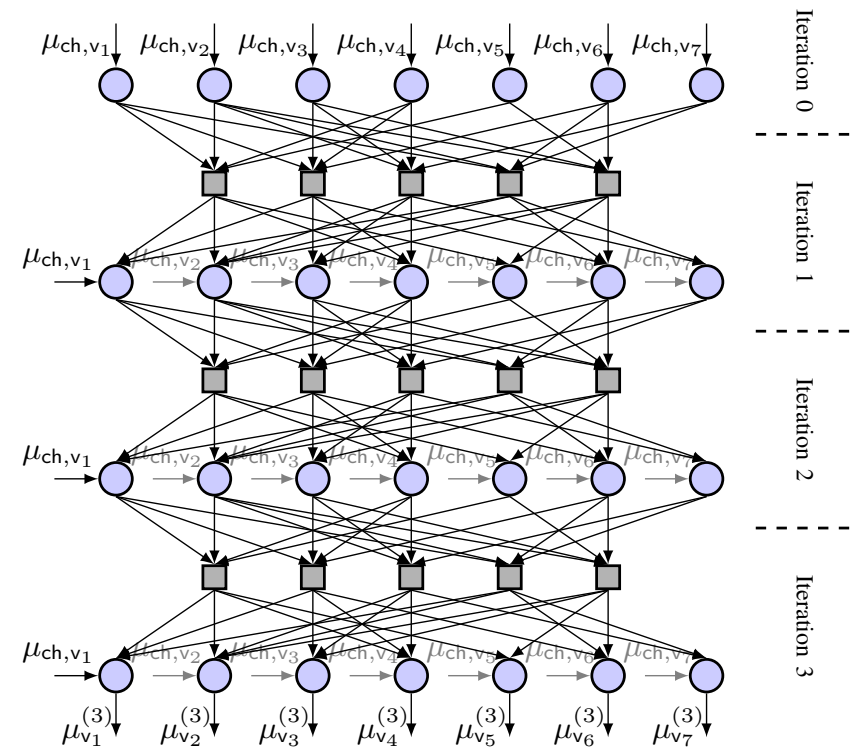

Fig. 1. Unrolled graph for three iterations where the blue circles denote VNs and the gray squares $\mathrm{CNs}$.

where $y_{i}$ is the channel output, $b_{i}$ is the transmitted bit, and $\sigma^{2}$ is the noise variance. The a posteriori log-likelihood ratio (LLR) in the $\ell$-th iteration is

$$
\mu_{\mathrm{v}_{i}}^{(\ell)}=\mu_{\mathrm{ch}, \mathrm{v}_{i}}+\sum_{\mathrm{c} \in \mathcal{N}\left(\mathrm{v}_{i}\right)} \mu_{\mathrm{c} \rightarrow \mathrm{v}_{i}}^{(\ell)} .
$$

A large contribution to the computational complexity stems from the $\mathrm{CN}$ update (2). A widely-used low-complexity approximation to (2) is the min-sum approximation [7]

$$
\mu_{c_{j} \rightarrow v_{i}}^{(\ell)}=\min _{v \in \mathcal{N}\left(c_{j}\right) \backslash v_{i}}\left|\mu_{v \rightarrow c_{j}}^{(\ell)}\right| \prod_{v \in \mathcal{N}\left(c_{j}\right) \backslash v_{i}} \operatorname{sign}\left(\mu_{v \rightarrow c_{j}}^{(\ell)}\right)
$$

where $\operatorname{sign}(\cdot)$ denotes the sign function. As this approximation tends to overestimate the magnitude of the messages, an additive $\mathrm{CN}$ - and iteration-dependent correction factor $\beta_{\mathrm{c}_{j}}^{(\ell)}$ is often introduced, leading to offset min-sum decoding [8]

$$
\begin{array}{r}
\mu_{c_{j} \rightarrow v_{i}}^{(\ell)}=\max \left(\min _{v \in \mathcal{N}\left(c_{j}\right) \backslash v_{i}}\left|\mu_{v \rightarrow c_{j}}^{(\ell)}\right|-\beta_{c_{j}}^{(\ell)}, 0\right) \\
\cdot \prod_{v \in \mathcal{N}\left(c_{j}\right) \backslash v_{i}} \operatorname{sign}\left(\mu_{v \rightarrow c_{j}}^{(\ell)}\right) .
\end{array}
$$

\section{A. Neural Belief Propagation}

For conventional BP, the decoder iterates between $\mathrm{VN}$ and $\mathrm{CN}$ updates by passing messages along the edges connecting VNs and CNs. For a given number of iterations $\ell_{\max }$, one can unroll the graph by stacking $\ell_{\max }$ copies of the Tanner graph. Consequently, the messages are passed in an unrolled fashion through the graph. This is illustrated in Fig. 1 for three iterations. One way to counteract the effect of short cycles on the performance of BP decoding for short linear block codes is to introduce weights for each edge of the unrolled Tanner graph [1], [2]. Due to the similarities of the weighted unrolled 
graph with a neural network, this is referred to as NBP. For NBP, the update rules (1) and (2) are modified to

$$
\mu_{\mathrm{v}_{i} \rightarrow \mathrm{c}_{j}}^{(\ell)}=w_{\mathrm{v}_{i} \rightarrow \mathrm{c}_{j}}^{(\ell)}\left(w_{\mathrm{ch}, \mathrm{v}_{i}}^{(\ell)} \mu_{\mathrm{ch}, \mathrm{v}_{i}}+\sum_{\mathrm{c} \in \mathcal{N}\left(\mathrm{v}_{i}\right) \backslash \mathrm{c}_{j}} \mu_{\mathrm{c} \rightarrow \mathrm{v}_{i}}^{(\ell)}\right)
$$

and

$$
\mu_{\mathrm{c}_{j} \rightarrow \mathrm{v}_{i}}^{(\ell)}=2 w_{\mathrm{c}_{j} \rightarrow \mathrm{v}_{i}}^{(\ell)} \tanh ^{-1}\left(\prod_{\mathrm{v} \in \mathcal{N}\left(\mathrm{c}_{j}\right) \backslash \mathrm{v}_{i}} \tanh \left(\frac{1}{2} \mu_{\mathrm{v} \rightarrow \mathrm{c}_{j}}^{(\ell)}\right)\right)
$$

where $w_{\mathrm{ch}, \mathrm{v}}^{(\ell)}, w_{\mathrm{v} \rightarrow \mathrm{c}}^{(\ell)}$, and $w_{\mathrm{c} \rightarrow \mathrm{v}}^{(\ell)}$, are the channel weights, the weights on the edges connecting VNs to $\mathrm{CNs}$, and the weights on the edges connecting CNs to VNs, respectively. The $a$ posteriori LLR in the $\ell$-th iteration is

$$
\mu_{\mathrm{v}_{i}}^{(\ell)}=w_{\mathrm{ch}, \mathrm{v}_{i}}^{(\ell)} \mu_{\mathrm{ch}, \mathrm{v}_{i}}+\sum_{\mathrm{c} \in \mathcal{N}\left(\mathrm{v}_{i}\right)} \mu_{\mathrm{c} \rightarrow \mathrm{v}_{i}}^{(\ell)} .
$$

In (4) and (5) the weights are untied over all nodes as well as over all iterations, i.e., each edge has an individual weight. In order to reduce complexity and storage requirements for NBP, the weights can also be tied. In [3], tying the weights temporally, i.e., over iterations, and spatially, i.e., all edges within a layer have the same weight, was explored. Note that setting all weights of an NBP decoder to one yields conventional BP decoding.

\section{B. Neural Offset Min-Sum Decoder}

Similar to the extension of conventional BP decoding to NBP decoding, the offset min-sum decoder can be extended to a NOMS decoder [5]. Instead of the CN- and iterationdependent offset $\beta_{\mathrm{c}}^{(\ell)}$, each edge emanating from a $\mathrm{CN}$ has its own offset,

$$
\begin{array}{r}
\mu_{\mathrm{c}_{j} \rightarrow \mathrm{v}_{i}}^{(\ell)}=\operatorname{ReLU}\left(\min _{\mathrm{v} \in \mathcal{N}\left(\mathrm{c}_{j}\right) \backslash \mathrm{v}_{i}}\left|\mu_{\mathrm{v} \rightarrow \mathrm{c}_{j}}^{(\ell)}\right|-\beta_{\mathrm{c}_{j} \rightarrow \mathrm{v}_{i}}^{(\ell)}\right) \\
\cdot \prod_{\mathrm{v} \in \mathcal{N}\left(\mathrm{c}_{j}\right) \backslash \mathrm{v}_{i}} \operatorname{sign}\left(\mu_{\mathrm{v} \rightarrow \mathrm{c}_{j}}^{(\ell)}\right)
\end{array}
$$

where $\operatorname{ReLU}(\cdot)=\max (\cdot, 0)$ denotes a rectified activation function commonly used in neural networks [16].

\section{Pruning-Based Neural Belief Propagation DECODERS}

Here we propose PB-NBP. The main idea is to consider NBP over the unrolled graph starting from a large, overcomplete parity-check matrix $\boldsymbol{H}_{\mathrm{oc}}$. We tie the weights for each $\mathrm{CN}$, i.e., $w_{\mathrm{c}_{j} \rightarrow \mathrm{v}_{i}}^{(\ell)}=w_{\mathrm{c}_{j}}^{(\ell)}$ for all $\mathrm{v}_{i} \in \mathcal{N}\left(\mathrm{c}_{j}\right)$,

$$
\mu_{\mathrm{c}_{j} \rightarrow \mathrm{v}_{i}}^{(\ell)}=2 w_{\mathrm{c}_{j}}^{(\ell)} \tanh ^{-1}\left(\prod_{\mathrm{v} \in \mathcal{N}\left(\mathrm{c}_{j}\right) \backslash \mathrm{v}_{i}} \tanh \left(\frac{1}{2} \mu_{\mathrm{v} \rightarrow \mathrm{c}_{j}}^{(\ell)}\right)\right) .
$$

We view the weights as an indication of the importance of the respective $\mathrm{CN}$ to the decoding and use them to prune the graph by successively removing CNs associated to low weights. The resulting graph potentially consists of a different set of CNs at each $\mathrm{CN}$ layer. This corresponds to selecting a (potentially) different set of parity-check equations from the overcomplete parity-check matrix in each iteration of BP decoding.

We first describe how we optimize the graph's weights and in a second step, we present the training procedure to prune the graph.

\section{A. Optimization of the Weights}

The decoding process can be seen as a classification task where the channel output is mapped to a valid codeword. This task consists of $2^{k}$ classes, one for each codeword. Training such a classification task is infeasible as the resulting decoder typically generalizes poorly to classes not contained in the training data [4]. Alternatively, one may consider a binary classification task for each of the $n$ bits. As a loss function, the average bitwise cross-entropy between the transmitted bits and the VN output LLRs of the final VN layer can be used [1], [2],

$$
\Gamma=-\frac{1}{n} \sum_{i=1}^{n} \log \left(o_{i}^{b_{i}}\left(1-o_{i}\right)^{1-b_{i}}\right)
$$

where $o_{i}$ is the estimate of the probability that the $i$-th bit after the final iteration is one,

$$
o_{i}=\frac{e^{-\mu_{v_{i}}^{\left(\ell_{\max }\right)}}}{1+e^{-\mu_{v_{i}}^{\left(\ell_{\max }\right)}}},
$$

and $\ell_{\max }$ denotes the number of decoding iterations. The optimization behavior can be improved by using a multiloss function [1], [2], where the overall loss is the average bitwise cross-entropy between the transmitted bits and the VN output LLR of each VN layer. The cross-entropy is well-suited for the bitwise classification task, but it does not necessarily result in a decoder with the lowest possible bit error rate. In fact, the bit error rate would be a more natural choice for the loss function. However, since the gradients would be zero almost everywhere, it is infeasible for optimization using gradient descent. Instead, [3] proposed a new loss function which can be regarded as soft bit error rate,

$$
\Gamma=\frac{1}{n} \sum_{i=1}^{n}\left(1-o_{i}\right)^{b_{i}} o_{i}^{1-b_{i}}
$$

It was empirically observed in [3] that minimizing this loss function also minimizes the bit error rate. Combining the soft bit error rate and a multiloss results in

$$
\begin{aligned}
& \tilde{\Gamma}=\frac{1}{\sum_{\ell} \eta^{\ell_{\max }-\ell}} \sum_{\ell=1}^{\ell_{\max }} \eta^{\ell_{\max }-\ell} \frac{1}{n} \sum_{i=1}^{n}\left(1-o_{i}^{(\ell)}\right)^{b_{i}}\left(o_{i}^{(\ell)}\right)^{1-b_{i}} \\
& \stackrel{(a)}{=} \frac{1}{\sum_{\ell} \eta^{\ell_{\max }-\ell}} \sum_{\ell=1}^{\ell_{\max }} \eta^{\ell_{\max }-\ell} \frac{1}{n} \sum_{i=1}^{n} o_{i}^{(\ell)}
\end{aligned}
$$

where $o_{i}^{(\ell)}$ is the estimate of the probability that the $i$-th bit is one after the $\ell$-th iteration, and $\eta \in(0 ; 1]$ determines the contribution of intermediate layers to the overall loss. Step (a) follows from the assumption that the all-zero codeword is transmitted, which is a valid assumption since the channel and the decoder are symmetric. The parameter $\eta$ is set close to one, i.e., all layers contribute equally to the loss, in the beginning of 


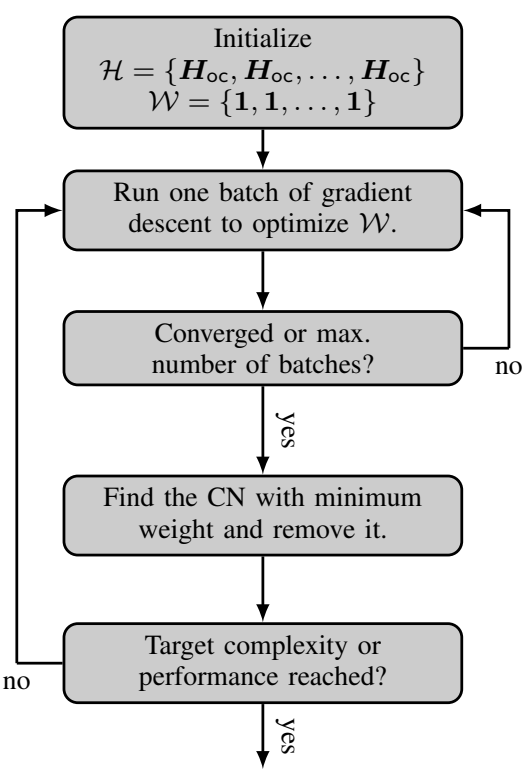

Fig. 2. Flowchart of the training process. 1 denotes the all-one matrix of appropriate size.

the training. This allows for gradients to efficiently propagate to earlier layers and hence improves convergence. However, this does not correspond to the desired, final behavior of the decoder where only the output of the final layer matters. Thus, the contribution of the intermediate layers is successively decreased during training by means of decreasing $\eta$. During the final stages of training, only the last layer will contribute to the loss, corresponding to the desired, final behavior [3].

\section{B. Training Procedure}

Consider NBP with VN update (4) and CN update (7) over the unrolled graph of $\boldsymbol{H}_{\mathrm{oc}}$ in which the weights are tied at the CNs, i.e., all messages emanating from a single $\mathrm{CN}$ c are weighted by the same weight $w_{\mathrm{c}}^{(\ell)}$. The magnitude of the weight $w_{c}^{(\ell)}$ can now be interpreted as a measure of how much $\mathrm{CN}$ c contributes to the decoding at iteration $\ell$. A large magnitude indicates high importance whereas a magnitude of zero indicates that the $\mathrm{CN}$ is irrelevant to the decoding process.

Let $\boldsymbol{H}_{\ell}$ be the parity-check matrix used for decoding in the $\ell$-th iteration and define $\mathcal{H}=\left\{\boldsymbol{H}_{1}, \ldots, \boldsymbol{H}_{\ell_{\max }}\right\}$. The set is initialized with the same large overcomplete matrix $\boldsymbol{H}_{\mathrm{oc}}$ for each iteration, i.e., $\mathcal{H}=\left\{\boldsymbol{H}_{\mathrm{oc}}, \ldots, \boldsymbol{H}_{\mathrm{oc}}\right\}$. Equivalently, we define a set of weights $\mathcal{W}$ and initialize all weights to one, i.e., we start with conventional BP. The weights in $\mathcal{W}$ are then optimized using the Adam optimizer [17] within the Tensorflow programming framework [18]. After the optimization has converged, we find the lowest $\mathrm{CN}$ weight $w_{\mathrm{c}}^{(\ell)}$ and set it to zero. This is equivalent to pruning the $\mathrm{CN}$ from the unrolled graph. As this may change the optimal value for the remaining weights, we rerun the training. We iterate between retraining and pruning $\mathrm{CNs}$ and monitor the loss. The optimization is stopped when the loss starts increasing. Empirically we observe that the loss is not monotone and that it is beneficial to allow the loss to increase slightly before stopping the pruning. Alternatively, one may further prune $\mathrm{CNs}$ to reach a desired number of parity-check equations

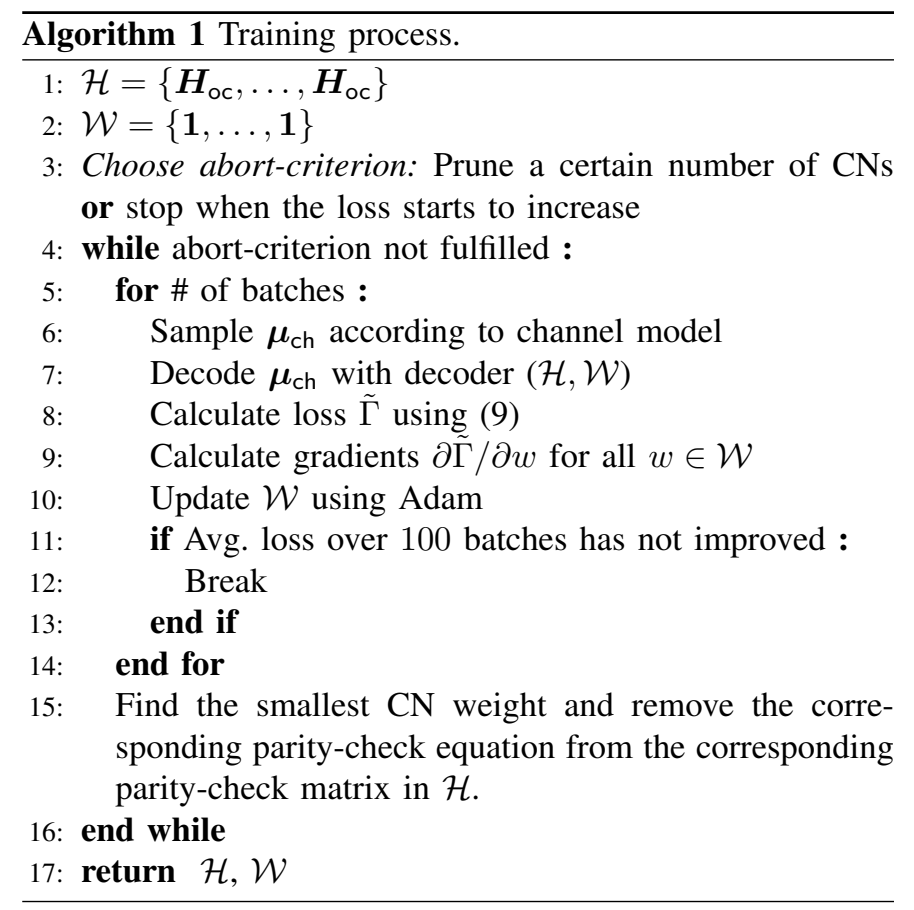

over all iterations, i.e., a given complexity, at the expense of a larger loss. The result of the optimization is a set of parity-check matrices $\mathcal{H}_{\mathrm{opt}}=\left\{\boldsymbol{H}_{1, \mathrm{opt}}, \ldots, \boldsymbol{H}_{\ell_{\text {max }}, \mathrm{opt}}\right\}$ and optimized weights $\mathcal{W}_{\text {opt }}$. The training process is illustrated in the flowchart of Fig. 2 and in Algorithm 1.

If the matrices used to initialize $\mathcal{H}$ are very large, this way of optimizing the parity-check matrices is potentially complex and slow. Empirically, we observed that it is possible to simultaneously prune more than one $\mathrm{CN}$ in the earlier stages of the pruning process without harming the final performance. This allows for a significant speed-up of the optimization. All results in this paper are achieved by only pruning a single $\mathrm{CN}$ per pruning step. An in-depth analysis of different pruning schedules is left for future work.

\section{Complexity Discussion}

In the following, we provide a high-level discussion of the decoding complexity. A thorough complexity analysis would require considering hardware implementation details such as data bus requirements, impact of the degree of parallelism and structure in the graph, etc. While this is out of the scope of the paper, we note that hardware constraints can potentially be incorporated in the training process (e.g., through a modified loss function), rendering our proposed decoders adaptable to different use cases.

On a high level, the $\mathrm{CN}$ update is the most complex operation in a BP decoder due to the evaluation of the tanh and inverse tanh functions. Hence, a commonly used measure for complexity is given by [19]

$$
\sum_{\ell} \bar{d}_{\mathrm{c}}^{(\ell)}\left|\mathcal{V}_{c}^{(\ell)}\right|
$$

where $\bar{d}_{c}^{(\ell)}$ denotes the average $\mathrm{CN}$ degree in the $\ell$-th iteration and $\mathcal{V}_{c}^{(\ell)}$ the set of active CNs in the $\ell$-th iteration. For conventional BP decoding, $\mathcal{V}_{\mathrm{c}}^{(\ell)}=\mathcal{V}_{\mathrm{c}}$ and $\bar{d}_{\mathrm{c}}^{(\ell)}=\bar{d}_{\mathrm{c}} \forall \ell$. 
The required memory is related to the parity-check matrix itself and the number of weights that need to be stored. Since the weights are real numbers as opposed to binary values for the edges, we quantify memory requirements with the number of weights. Furthermore, the weights require additional multiplications.

Following this discussion, we define three decoders of different complexity.

- PB-NBP decoder $\mathcal{D}_{1}$ : It uses the result from the optimization directly, i.e., $\mathcal{H}_{\text {opt }}$ and $\mathcal{W}_{\text {opt }}$.

- PB-NBP decoder $\mathcal{D}_{2}$ : It uses the optimized set of paritycheck matrices, i.e., $\mathcal{H}_{\mathrm{opt}}$, but sets all weights to one, i.e., neglects $\mathcal{W}_{\text {opt }}$.

- PB-NBP decoder $\mathcal{D}_{3}$ : It uses the optimized set of paritycheck matrices, i.e., $\mathcal{H}_{\text {opt }}$, and additionally untied optimized weights over all iterations and edges. It is important to note that to obtain the untied weights, an extra training step with untied weights is required to obtain $\mathcal{W}_{\text {opt }}$.

All three decoders require the same number of $\mathrm{CN}$ evaluations as they operate on the same set of parity-check matrices $\mathcal{H}_{\text {opt }}$. However, they differ in the required memory. PB-NBP decoder $\mathcal{D}_{3}$ needs to store one weight per edge, whereas PB-NBP decoder $\mathcal{D}_{2}$ does not need to store any weights. PB-NBP decoder $\mathcal{D}_{1}$ needs to store one weight per channel message and per edge emanating from a VN but only one weight per $\mathrm{CN}$ and hence is of lower complexity compared to PB-NBP $\mathcal{D}_{3}$ but is more complex than PB-NBP $\mathcal{D}_{2}$.

Remark: We note that in terms of performance, pruning a $\mathrm{CN}$ is equivalent to setting the weights of all outgoing edges from a $\mathrm{CN}$ to zero. Hence, an NBP decoder could in theory be trained to yield exactly the same performance as a PB-NBP decoder. However, the NBP decoder would have a higher complexity than the PB-NBP decoder as the number of $\mathrm{CN}$ evaluations would not be reduced. Furthermore, empirically, we do not observe that weights converge to zero for NBP. In this light, pruning allows for a reduction in complexity as well as facilitates training. This is in line with the results in [20], where the authors observed that by iteratively pruning and retraining a vanilla neural network, the network achieves higher accuracy and converges faster than the original, unpruned network or a network pruned using a single iteration.

\section{Pruning-Based Neural Offset Min-Sum Decoder}

We extend the pruning concept to NOMS decoding. The same procedure as the one described in the previous section can be applied to find the optimal set of parity-check matrices and weights for PB-NOMS. However, since both NBP and NOMS are iterative algorithms differing only in the $\mathrm{CN}$ update, we hypothesize that the same set of parity-check matrices optimized for NBP performs well for NOMS. This is confirmed empirically by comparing the performance of a PBNOMS decoder with parity-check matrices optimized using NBP and a PB-NOMS decoder with parity-check matrices optimized using NOMS.

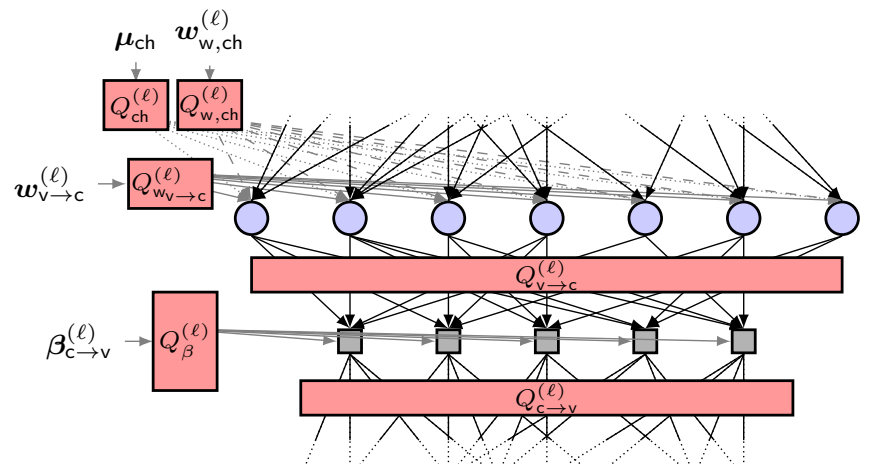

Fig. 3. Block diagram of the $\ell$-th $\mathrm{VN}$ and $\mathrm{CN}$ layer of the unrolled (PB-)NOMS decoder with quantization. The blue circles denote VNs, the gray squares CNs, $\boldsymbol{\mu}_{\mathrm{ch}}$ the vector containing all $n$ channel messages, $\boldsymbol{w}_{\mathrm{v} \rightarrow c}^{(\ell)}$ and $\boldsymbol{w}_{\mathrm{ch}}^{(\ell)}$ the vectors containing the respective weights, and $\boldsymbol{\beta}_{\mathrm{c} \rightarrow \mathrm{v}}^{(\ell)}$ the vector containing the offsets.

\section{Quantization of The PB-NOMS Decoder}

We consider quantization of the channel output, the messages between the layers, and the weights and offsets of the PB-NOMS decoder. In particular, we consider a joint optimization of the quantization, the weights, and the offsets. To this end, we first define a symmetric mid-tread quantizer as a piecewise constant function $Q(x)$ with $2^{n_{q}}-1$ quantization levels $\mathcal{Q}=\left\{q_{0}=0, \pm q_{1}, \ldots, \pm q_{2^{\left(n_{q}-1\right)}-1}\right\}$ and thresholds $\mathcal{T}=\left\{t_{1}, \ldots, t_{2^{\left(n_{q}-1\right)}-1}\right\}$,

$$
Q(x)=\left\{\begin{array}{cc}
0 & |x|<t_{1}, \\
\operatorname{sign}(x) q_{1} & t_{1} \leq|x|<t_{2}, \\
\vdots & \vdots \\
\operatorname{sign}(x) q_{i} & t_{i} \leq|x|<t_{i+1}, \\
\vdots & \vdots \\
\operatorname{sign}(x) q_{2^{\left(n_{q}-1\right)}-1} & t_{2^{\left(n_{q}-1\right)}-1} \leq|x| .
\end{array}\right.
$$

The gradients of $Q(x)$ with respect to $x$ are zero except at the thresholds. During training in the back-propagation phase, these zero-gradients would cause most gradients in the network to be zero and hence prohibit the training to converge to a meaningful solution. Using the gradient of a surrogate function, referred to as STE, overcomes this issue [15]. In this work, we simply pass through the gradients with respect to $x$, i.e., $\partial Q(x) / \partial x=1$. By letting $t_{i}=\left(q_{i}+q_{i+1}\right) / 2$, the gradients with respect to the quantization levels are

$$
\frac{\partial Q(x)}{\partial q_{i}}=\left\{\begin{array}{cc}
\operatorname{sign}(x) & t_{i} \leq|x|<t_{i+1} \\
0 & \text { else }
\end{array}\right.
$$

and

$$
\frac{\partial Q(x)}{\partial q_{2^{n_{q}-1}-1}}=\left\{\begin{array}{cc}
\operatorname{sign}(x) & t_{2^{\left(n_{q}-1\right)}-1} \leq|x|, \\
0 & \text { else }
\end{array}\right.
$$

for $i \in\left\{1, \ldots 2^{n_{q}-1}-2\right\}$.

Denote now as $Q_{\mathrm{ch}}^{(\ell)}, Q_{\mathrm{c} \rightarrow \mathrm{v}}^{(\ell)}, Q_{\mathrm{v} \rightarrow \mathrm{c}}^{(\ell)}, Q_{\mathrm{w}_{\mathrm{v}} \rightarrow \mathrm{c}}^{(\ell)}$, and $Q_{\beta}^{(\ell)}$ the quantizers in the $\ell$-th iteration for the channel messages, the $\mathrm{CN}$-to-VN messages, the VN-to-CN messages, the VN-to-CN weights, and the offsets corresponding to the $\mathrm{CN}$ update (see (6)), respectively.

In Fig. 3, we show a block diagram of the $\ell$-th $\mathrm{VN}$ and VN layer of a (PB-)NOMS decoder. While the forward and 


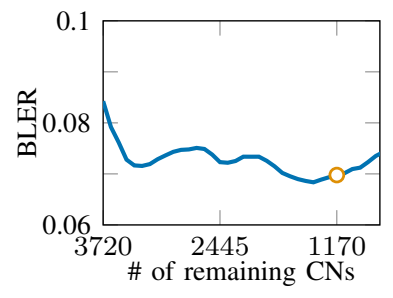

(a) BLER during the iterative pruning process. The circle marks the number of $\mathrm{CNs}$ of PB-NBP $\mathcal{D}_{1}, \mathcal{D}_{2}$, and $\mathcal{D}_{3}$.

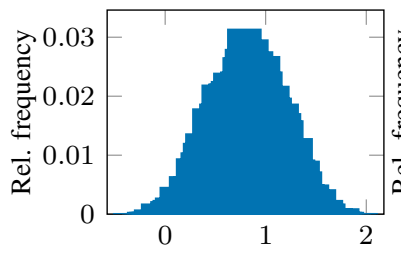

(c) Histogram of the $\mathrm{CN}$ weights in the final iteration.

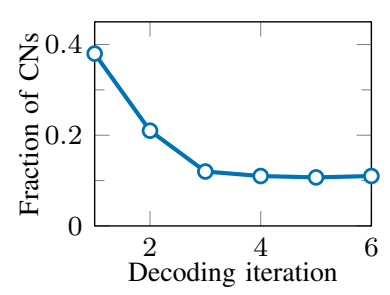

(b) Fraction of the remaining the respective $\mathrm{CN}$ layer.

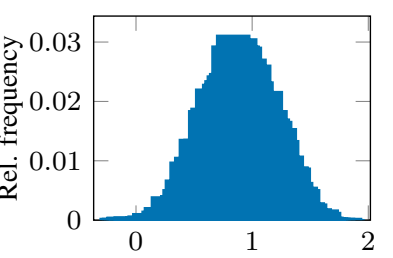

(d) Histogram of the $\mathrm{VN}$ weights in the final iteration. CNs after pruning in

Fig. 4. Results from the training process for the $\operatorname{RM}(2,5)$ code.

backward pass of the training of the quantized PB-NOMS decoder take into account the quantization, the update of trainable weights during training is performed using floating point precision. To emphasize this, we depict the weights, quantized by its associated quantizer, as an input to the respective node. Further, while the quantizers are untied over the layers, i.e., each layer has its own quantization levels and thresholds, we tie the number of bits over the layers. We denote the number of bits for quantizing the channel messages as $q_{\mathrm{ch}}$, the number of bits for messages passed between nodes as $q_{\mathrm{m}}$, and the number of bits for all the weights and offsets as $q_{\mathrm{w}}$.

\section{NumERicAl RESUlts}

We numerically evaluate the performance of the proposed PB-NBP and PB-NOMS decoders for RM codes, a short lowdensity parity-check (LDPC) code, and a polar code. As a benchmark we consider ML decoding and we compare the performance of the proposed decoders to NBP [1], NOMS [5], and MBBP decoding [12] (referred to as MBBP-NX-S in [12]). The hyperparameters are provided in the appendix and the source code is available online [21].

\section{A. Reed-Muller Code RM(2,5)}

For the $\operatorname{RM}(2,5)$ code of length $n=32$ and dimension $k=16$, we consider the overcomplete parity-check matrix $\boldsymbol{H}_{\mathrm{oc}}$ containing all 620 parity-check equations of minimum weight and apply the training procedure described in Section III-B to it. We fix the number of iterations to six. Hence, without pruning $620 \mathrm{CNs}$ need to be evaluated per $\mathrm{CN}$ layer, which leads to a total of $3720 \mathrm{CNs}$ that need to be evaluated. Note that since $\boldsymbol{H}_{\mathrm{oc}}$ has a regular $\mathrm{CN}$ degree and pruning does not affect the $\mathrm{CN}$ degree, we can neglect the average $\mathrm{CN}$ degree in (10) for the complexity discussion. The optimization is stopped when the loss starts to increase, which corresponds to keeping $31 \%$ of the CNs of the unrolled graph. This is shown in Fig. 4(a), where the block error rate (BLER) during

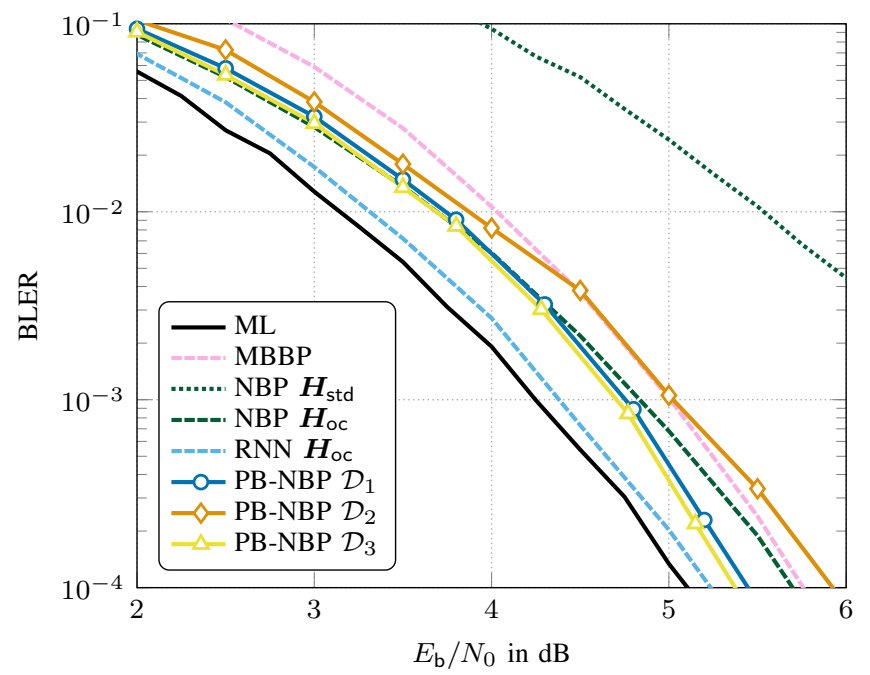

Fig. 5. BLER results for the $\mathrm{RM}(2,5)$ code with (PB-)NBP decoding.

TABLE I

COMPLEXITY OF THE RM $(2,5)$ CODE. IN PARENTHESES THE FRACTION OF THE NUMBER OF CNS AND WEIGHTS COMPARED TO NBP WITH $\boldsymbol{H}_{\text {OC }}$ (DENOTED BY"1.0").

\begin{tabular}{lrlrl}
\hline & \multicolumn{2}{c}{ \# of CNs } & \multicolumn{2}{c}{ \# of weights and offsets } \\
\hline NBP $\boldsymbol{H}_{\text {oc }}$ & 3720 & $(1.0)$ & 64704 & $(1.0)$ \\
RNN $\boldsymbol{H}_{\text {oc }}$ & 3720 & $(1.0)$ & 10144 & $(0.157)$ \\
NBP $\boldsymbol{H}_{\text {std }}$ & 96 & $(0.026)$ & 328 & $(0.005)$ \\
MBBP RM $(2,5)$ & 1440 & $(0.387)$ & 0 & $(0.0)$ \\
\hline PB-NBP D D & 1170 & $(0.315)$ & 10754 & $(0.166)$ \\
PB-NBP D D & 1170 & $(0.315)$ & 0 & $(0.0)$ \\
PB-NBP D & 1170 & $(0.315)$ & 18944 & $(0.293)$ \\
PB-NBP Random & 1170 & $(0.315)$ & 10754 & $(0.166)$ \\
PB-NOMS & 1170 & $(0.315)$ & 9360 & $(0.145)$ \\
\hline
\end{tabular}

the iterative pruning process is shown as a function of the number of remaining CNs. The marker highlights where the pruning is stopped. Note that this does not correspond to the minimum of the BLER as training is only stopped after the loss started to increase (see also Section III-B). To investigate the behavior of pruning, we are interested in how many CNs are pruned in each CN layer. In Fig. 4(b), we plot the distribution of surviving $\mathrm{CNs}$ after pruning across $\mathrm{CN}$ layers. We observe that in the first $\mathrm{CN}$ layer, about $40 \%$ of all surviving $\mathrm{CNs}$ are used for decoding. In later $\mathrm{CN}$ layers, the number of $\mathrm{CNs}$ decreases significantly. This observation furthermore justifies the use of a low number of iterations. In Fig. 4(c) and 4(d), we plot the histograms of the $\mathrm{CN}$ and $\mathrm{VN}$ weights in the final iteration.

In Fig. 5, we plot the BLER as a function of $E_{\mathrm{b}} / N_{0}$. The PB-NBP decoder $\mathcal{D}_{1}$ performs $0.38 \mathrm{~dB}$ away from the $\mathrm{ML}$ decoder at a BLER of $10^{-4}$. Removing the weights (PB-NBP $\mathcal{D}_{2}$ ), results in a penalty of $0.48 \mathrm{~dB}$. Untying the weights in the CNs (PB-NBP $\mathcal{D}_{3}$ ) results in an additional gain of $0.047 \mathrm{~dB}$ with respect to PB-NBP $\mathcal{D}_{1}$.

Both PB-NBP $\mathcal{D}_{1}$ and $\mathcal{D}_{3}$ outperform NBP with $\boldsymbol{H}_{\mathrm{oc}}$ containing the 620 parity-check equations of minimum weight, as well as MBBP [12] with 15 randomly chosen parity-check matrices. Furthermore, the proposed PB-NBP decoders are less complex than NBP with $\boldsymbol{H}_{\text {oc }}$ and MBBP, requiring $68 \%$ and $7 \%$ less $\mathrm{CN}$ evaluations than $\mathrm{NBP}$ and $\mathrm{MBBP}$, respectively. 


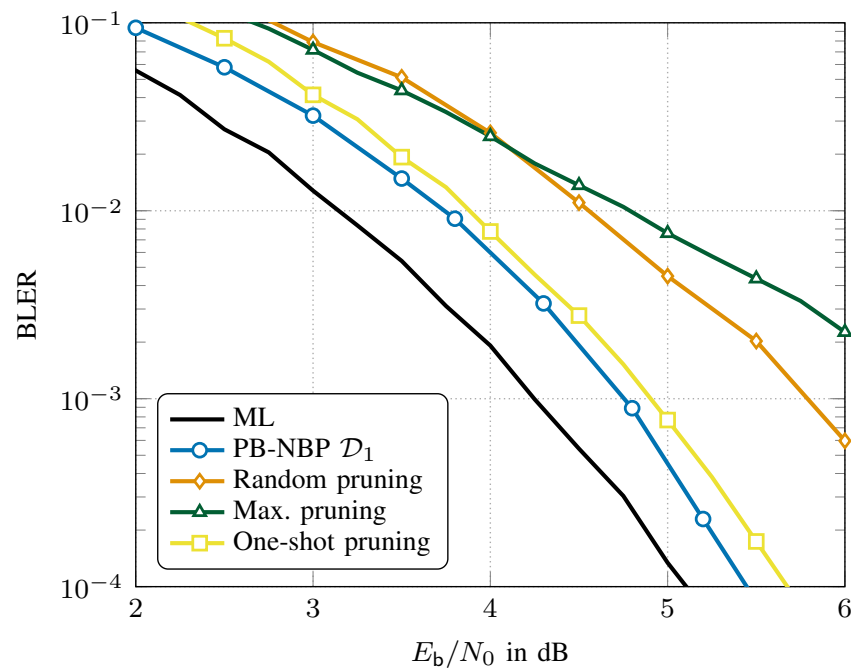

Fig. 6. Comparison of different pruning strategies for the $\mathrm{RM}(2,5)$ code.

PB-NBP $\mathcal{D}_{2}$ performs slightly worse than NBP, but entails the lowest complexity as no weights need to be stored. As a further comparison, we also plot the performance of a recurrent neural network (RNN)-based decoder [2] using $\boldsymbol{H}_{\mathrm{oc}}$. The RNN-based decoder slightly outperforms PB-NBP $\mathcal{D}_{1}$ and PB-NBP $\mathcal{D}_{2}$ at the cost of increased complexity by a factor of three. NBP with a standard parity-check matrix with no redundant rows (i.e., containing 16 parity-check equations) is clearly not competitive. The PB-NBP decoders also require significantly less weights than the NBP decoder with $\boldsymbol{H}_{\text {oc }}$. The decoding complexity of the decoders in Fig. 5 is reported in Table I. In parentheses, we display the complexity normalized by that of NBP with $\boldsymbol{H}_{\text {oc }}$.

To assess the effectiveness of our pruning strategy, we also consider the scenarios where we randomly prune $\mathrm{CNs}$ (random pruning), where we prune the $\mathrm{CN}$ associated with the largest weight (referred to as maximum pruning), and all CNs in a single step (one-shot pruning). As it can be observed in Fig. 6, the performance of random and maximum pruning is clearly not competitive. One-shot pruning exhibits a loss of about $0.1 \mathrm{~dB}$ over our proposed pruning method. While one-shot pruning may speed up the training process, it is important to note that training is done offline and the final decoders have the same complexity. Furthermore, one-shot pruning requires that the final number of CNs is known a priori.

The BLER of the PB-NOMS decoder with $q_{\mathrm{m}}$ bits for the messages and $q_{\mathrm{w}}$ bits for the weights is depicted in Fig. 7, where $\infty$ bits denotes full precision floating point. We further set $q_{\mathrm{ch}}=q_{\mathrm{m}}$. Floating point PB-NOMS decoding suffers from a $0.25 \mathrm{~dB}$ degradation over PB-NBP decoding. Further quantizing the PB-NOMS decoder increases this gap. We observe that while for $q_{\mathrm{w}}=5,3$ bits for the channel output and internal messages appears to be sufficient, in the case of $q_{\mathrm{w}}=3$ using 3 bits for the channel output and internal messages leads to a significant performance degradation with respect to floating point messages. The PB-NOMS and the PBNBP decoders require the same number of $\mathrm{CN}$ evaluations and weights. However, the PB-NOMS decoder is less complex, due to its simplified $\mathrm{CN}$ update.

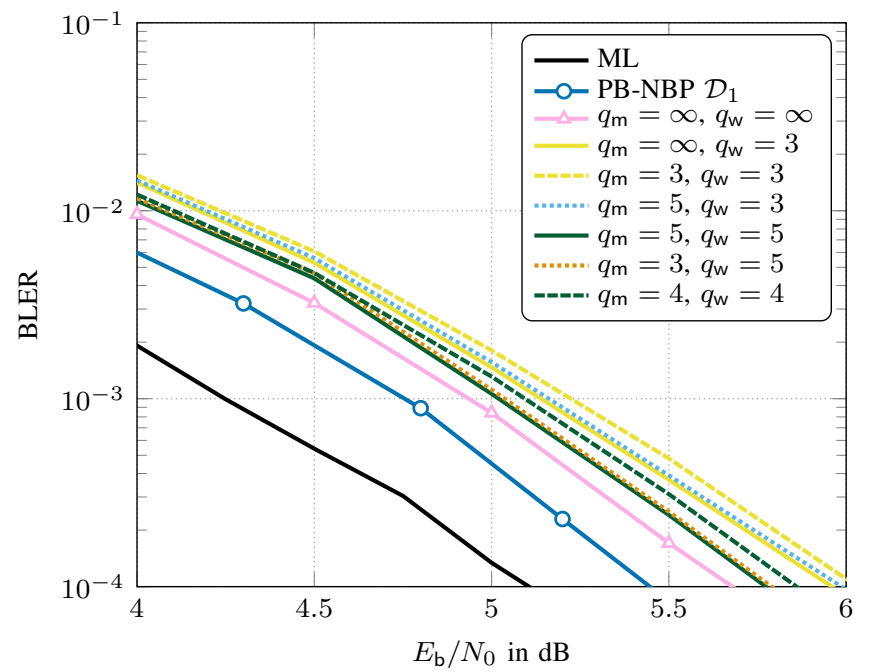

Fig. 7. BLER results for the $\operatorname{RM}(2,5)$ code with PB-NOMS decoding and quantization.

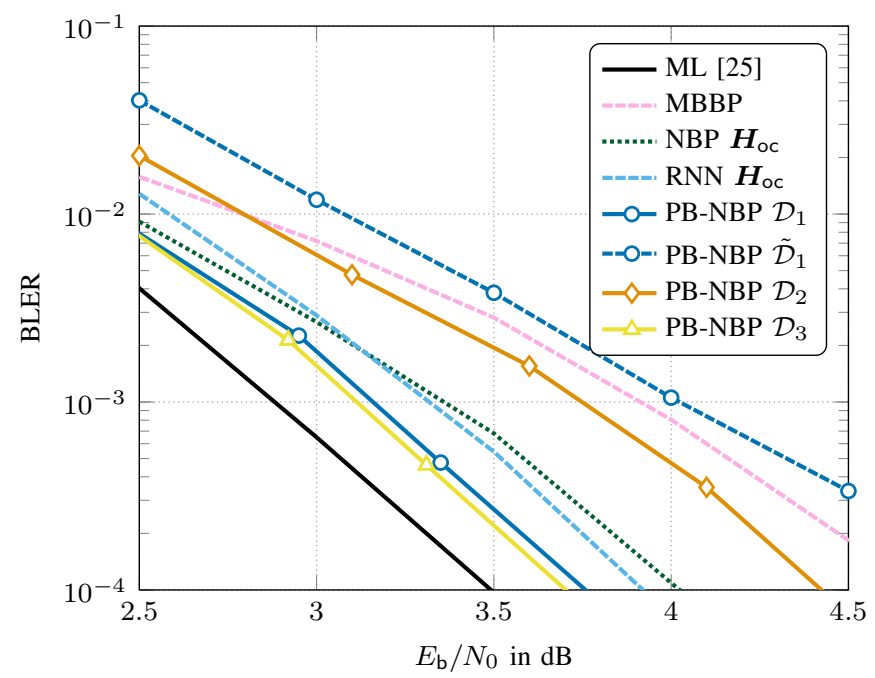

Fig. 8. BLER results for the $\mathrm{RM}(3,7)$ code with (PB-)NBP decoding.

\section{B. Reed-Muller Code RM(3,7)}

For the $\operatorname{RM}(3,7)$ code of code length $n=128$ and dimension $k=64$, we select as the overcomplete paritycheck matrix $\boldsymbol{H}_{\text {oc }}$ the matrix containing 70000 out of 94488 randomly selected parity-check equations of minimum weight. Since in the initial training phase $\mathrm{CNs}$ are removed in an almost random fashion, choosing a large random subset of all minimum-weight parity-check equations to initialize the training does not harm the optimization. To investigate the effect of the size of the random subsets of minimum-weight parity-check equations, we also consider the case where only a small, random subset, containing 9448 of all parity-check equations of minimum weight are selected for $\boldsymbol{H}_{\mathrm{oc}}$ and denote this decoder as PB-NBP $\tilde{\mathcal{D}}_{1}$. Again, we fix the number of iterations to six. In Fig. 8, we plot the BLER for the PB-NBP decoders $\mathcal{D}_{1}, \tilde{\mathcal{D}}_{1}, \mathcal{D}_{2}$, and $\mathcal{D}_{3}$ and compare the performance to that of NBP and MBBP. Decoder PB-NBP $\mathcal{D}_{1}$ performs $0.27 \mathrm{~dB}$ from the ML decoder and improves upon NBP with $\boldsymbol{H}_{\text {oc }}$ by $0.28 \mathrm{~dB}$. Removing the weights results in a degradation of $0.47 \mathrm{~dB}$ for decoder PB-NBP $\mathcal{D}_{2}$ with respect 
TABLE II

COMPLEXITY OF THE RM $(3,7)$ CODE. IN PARENTHESES THE FRACTION OF THE NUMBER OF CNS AND WEIGHTS COMPARED TO NBP WITH $\boldsymbol{H}_{\text {oc }}$ (DENOTED BY "1.0").

\begin{tabular}{lrlrl}
\hline & \multicolumn{2}{c}{ \# of CNs } & \# of weights and offsets \\
\hline NBP $\boldsymbol{H}_{\text {oc }}$ & 566928 & $(1.0)$ & 19654400 & $(1.0)$ \\
RNN $\boldsymbol{H}_{\text {oc }}$ & 566928 & $(1.0)$ & 3023744 & $(0.154)$ \\
MBBP & 23440 & $(0.0413)$ & 0 & $(0.0)$ \\
\hline PB-NBP $\mathcal{D}_{1}$ & 19842 & $(0.0349)$ & 2342252 & $(0.119)$ \\
PB-NBP $\mathcal{D}_{2}$ & 19842 & $(0.0349)$ & 0 & $(0.0)$ \\
PB-NBP $\mathcal{D}_{3}$ & 19842 & $(0.0349)$ & 4128032 & $(0.21)$ \\
PB-NBP $\tilde{\mathcal{D}}_{1}$ & 19842 & $(0.0349)$ & 2342252 & $(0.119)$ \\
PB-NOMS & 19842 & $(0.0349)$ & 1904832 & $(0.097)$ \\
\hline
\end{tabular}

to PB-NBP $\mathcal{D}_{1}$. On the other hand, untying the weights results in a gain of $0.02 \mathrm{~dB}$ for decoder PB-NBP $\mathcal{D}_{3}$.

Decoders PB-NBP $\mathcal{D}_{1}$, PB-NBP $\mathcal{D}_{2}$, and PB-NBP $\mathcal{D}_{3}$, require only $3.49 \%$ of the CNs and at most $21 \%$ of the weights compared to the NBP decoder with $\boldsymbol{H}_{\mathrm{oc}}$ while showing a performance gain of $0.28 \mathrm{~dB}$ for decoder PB-NBP $\mathcal{D}_{3}$. The RNN-based decoder with $\boldsymbol{H}_{\mathrm{oc}}$ requires a similar number of weights as the PB-NBP decoder $\mathcal{D}_{3}$, but is significantly more complex as it has the same number of $\mathrm{CNs}$ as the NBP decoder. However, it offers a worse performance than PB-NBP $\mathcal{D}_{1}$ and $\mathcal{D}_{3}$. As for the $\operatorname{RM}(2,5)$ code, NBP using the nonredundant parity-check matrix with $64 \mathrm{CNs}$ is not competitive (curve omitted for better readability). The complexities are reported in Table II. As for the $\operatorname{RM}(2,5)$ code, we omit the scaling by the average constant $\mathrm{CN}$ degree.

Decoder PB-NBP $\tilde{\mathcal{D}}_{1}$ demonstrates the effect of using only a small subset of all parity-check equations of minimum weight as the overcomplete parity-check matrix $\boldsymbol{H}_{\mathrm{oc}}$. The decoder is pruned to the same complexity as PB-NBP $\mathcal{D}_{1}$. As randomly selecting a small subset of parity-check equations essentially corresponds to randomly pruning CNs, we observe the same performance degradation as for the random pruning in the case of the $\operatorname{RM}(2,5)$ code.

In Fig. 9, we report BLER results for the quantized and pruned NOMS decoders. Once again, we set $q_{\mathrm{ch}}=q_{\mathrm{m}}$. At a BLER of $10^{-4}$, with $q_{\mathrm{m}}=q_{\mathrm{w}}=5$, we perform $0.5 \mathrm{~dB}$ from ML and with only 3 bits, we perform $0.8 \mathrm{~dB}$ from ML. In the figure we also compare the performance of the joint optimization of the quantizers, weights, and offsets to the that of two common approaches to quantization in neural networks-post-training quantization and quantization-aware training. For post-training quantization, the decoder is trained using floating point precision and the quantizer is added after the training is completed. To this end, we use Tensorflow's built-in quantizer, i.e., a uniform quantizer. The clipping range for the messages is set to \pm 8 . The quantizer of the weights and offsets is clipped to the range of the weights and offsets in the respective layer. From Fig. 9, we notice that this way of quantizing is clearly not competitive. One reason for this is that the weights and offsets of the decoder may not be optimal once the quantizers are added, as quantization distorts both weights and offsets. Furthermore, a different set of weights and offsets may be able to (partially) compensate for the performance degradation due to the quantized messages. Incorporating the quantizer into the training, referred to as quantization-aware

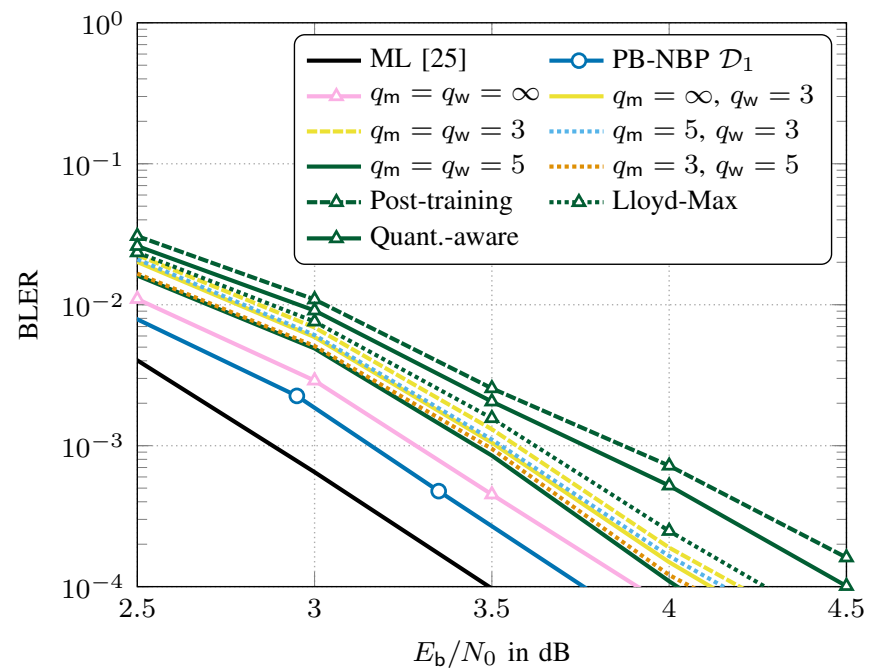

Fig. 9. BLER results for the $\operatorname{RM}(3,7)$ code with PB-NOMS decoding and quantization. For post-training quantization and quantization-aware training, $q_{\mathrm{m}}=q_{\mathrm{ch}}=q_{\mathrm{w}}=3$ is used.

TABLE III

COMPLEXITY OF THE CCSDS LDPC CODE. IN PARENTHESES THE FRACTION OF THE NUMBER OF CNS COMPARED TO CONVENTIONAL BP WITH 100 DECODING ITERATIONS (DENOTED BY "1.0").

\begin{tabular}{lrlr}
\hline & \multicolumn{2}{c}{ Complexity $(10)$} & \# of weights and offsets \\
\hline BP, 100 iterations & 51200 & $(1.0)$ & 0 \\
BP, 25 iterations & 12800 & $(0.25)$ & 0 \\
NBP $\boldsymbol{H}_{\text {std }}$ & 768 & $(0.015)$ & 13696 \\
\hline PB-NBP $\mathcal{D}_{1}$ & 25920 & $(0.506)$ & 28416 \\
PB-NOMS & 25920 & $(0.506)$ & 25920 \\
\hline
\end{tabular}

training, overcomes this. Once again, we use Tensorflow's built-in quantizer for this, clip the messages to \pm 8 and the weights and offsets to the range of the weights and offsets in the respective layer. Even though quantization-aware training improves upon post-training quantization, it is limited by the initial choice of using a uniform quantizer and the clipping range for the messages. For post-training quantization, a quantizer optimized using the Lloyd-Max algorithm [22], [23] improves significantly over the uniform quantizers. However, as the weights and offsets are potentially suboptimal, a small degradation to the proposed joint optimization remains.

\section{Low-Density Parity-Check Code}

We consider the CCSDS LDPC code of length 128 and rate 0.5 as defined in [24]. It has $\mathrm{CN}$ degree 8 and half the VNs have degree 3 and half have degree 5 . The code has minimum Hamming distance 14. We consider conventional BP decoding with 25 iterations, corresponding to $1600 \mathrm{CN}$ updates.

We let the overcomplete parity-check matrix $\boldsymbol{H}_{\text {oc }}$ contain 10000 randomly chosen parity-check equations of Hamming weight up to twenty. This causes CNs of different degrees and hence $\boldsymbol{H}_{\mathrm{oc}}$ has irregular $\mathrm{CN}$ degree. We then design the PBNBP decoder with six iterations and the same number of $\mathrm{CN}$ evaluations as for conventional BP decoding with 25 iterations. Note that as the parity-check matrices of the BP and PB-NBP decoders are of irregular $\mathrm{CN}$ degree, the complexity is given by (10). 


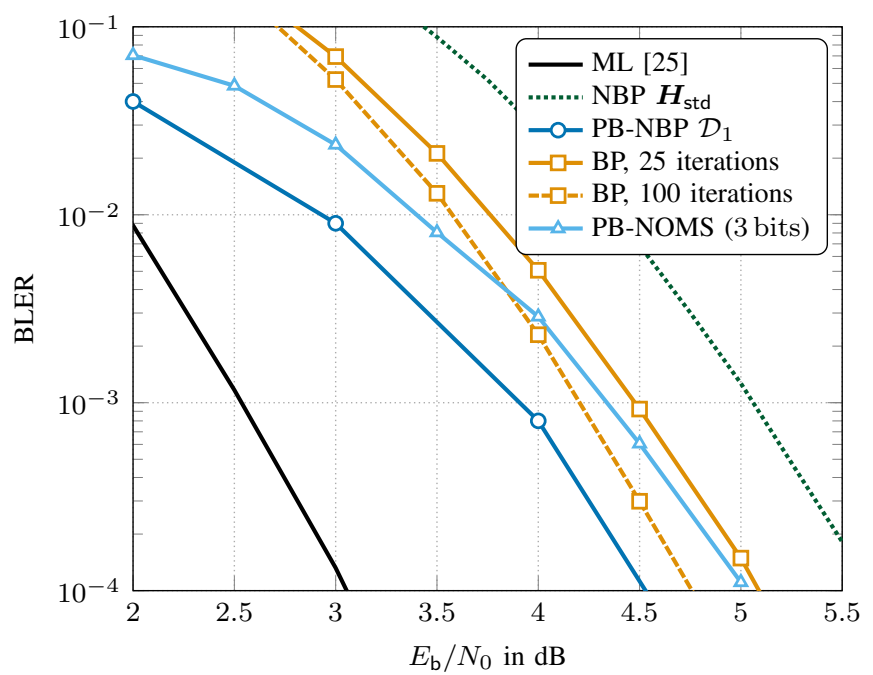

Fig. 10. BLER results for the LDPC code.

TABLE IV

COMPLEXITY OF THE POLAR CODE. IN PARENTHESES THE FRACTION OF THE NUMBER OF CNS AND WEIGHTS COMPARED TO NBP WITH $\boldsymbol{H}_{\text {oc }}$ (DENOTED BY "1.0").

\begin{tabular}{lrlrl}
\hline & \multicolumn{2}{c}{ \# of CNs } & \multicolumn{2}{c}{ \# of weights and offsets } \\
\hline NBP $\boldsymbol{H}_{\text {oc }}$ & 589824 & $(1.0)$ & 20448128 & $(1.0)$ \\
NBP $\boldsymbol{H}_{\text {std }}$ & 384 & $(0.00065)$ & 14208 & $(0.00069)$ \\
\hline PB-NBP $\mathcal{D}_{1}$ & 19842 & $(0.033)$ & 2342252 & $(0.115)$ \\
PB-NBP $\mathcal{D}_{2}$ & 19842 & $(0.033)$ & 0 & $(0.0)$ \\
PB-NOMS & 19842 & $(0.033)$ & 1904832 & $(0.064)$ \\
\hline
\end{tabular}

The BLER results are given in Fig. 10. PB-NBP decoder $\mathcal{D}_{1}$ outperforms conventional BP with 25 iterations by approximately $0.6 \mathrm{~dB}$. Allowing 100 iterations for conventional $\mathrm{BP}$ reduces the gain to $0.2 \mathrm{~dB}$. However, conventional $\mathrm{BP}$ with 100 iterations requires approximately twice the complexity of decoder PB-NBP $\mathcal{D}_{1}$. NBP with $64 \mathrm{CNs}$ is again not competitive. The complexity of the different decoders is reported in Table III. We remark that the decoding complexity of BP decoding does not take into account potential early stopping of the decoder. However, early stopping may also be used in the proposed PB-NBP decoders. The effects of quantization are similar to the ones observed for the ReedMuller codes. Quantizing messages, channel output, weights, and offsets with 3 bits causes a degradation of $0.3 \mathrm{~dB}$ over PB-NBP $\mathcal{D}_{1}$, which corresponds to a performance of $1.8 \mathrm{~dB}$ from ML decoding.

\section{Polar Code}

We finally consider a polar code of length $n=128$ and rate 0.5 defined in [25]. It has minimum Hamming distance 8 and its dual code has minimum Hamming distance 16 . Following [26], we find all 98304 codewords of the dual code of minimum weight. As for the $\operatorname{RM}(3,7)$ code, $\boldsymbol{H}_{\text {oc }}$ used for the optimization process contains 70000 randomlyselected minimum-weight parity-check equations to reduce the complexity of the training. As a target complexity, we choose the same complexity as for the $\operatorname{RM}(3,7)$ code. The final complexities are reported in Table IV. Once again, we omit the scaling by the average constant $\mathrm{CN}$ degree.

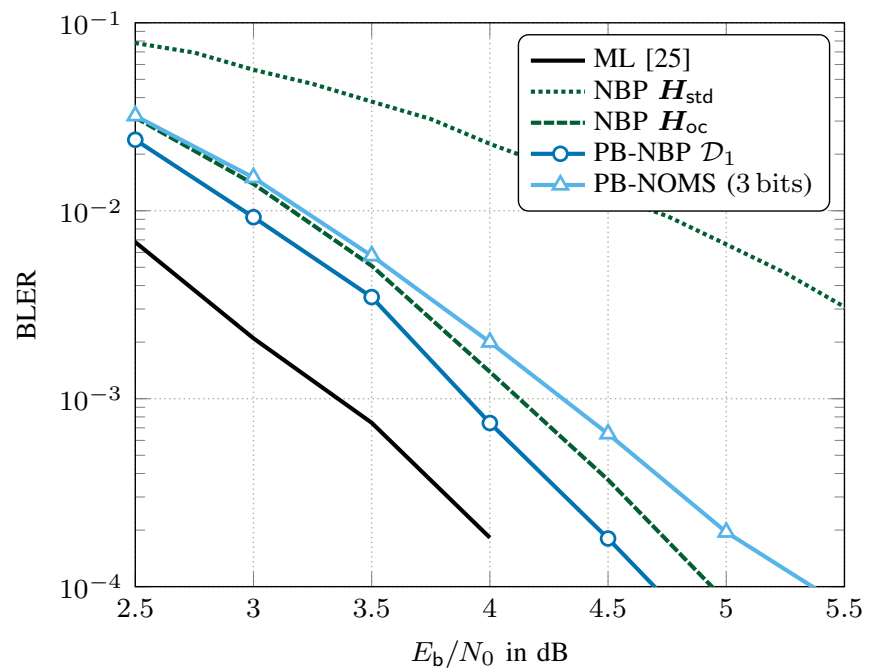

Fig. 11. BLER results for the polar code.

In Fig. 11, we plot the BLER as a function of $E_{\mathrm{b}} / N_{0}$. We observe a similar behavior to that of the RM codes. The PB-NBP decoder $\mathcal{D}_{1}$ performs $0.5 \mathrm{~dB}$ from $\mathrm{ML}$ and outperforms NBP with $\boldsymbol{H}_{\mathrm{oc}}$ while only requiring $3.3 \%$ of its CNs evaluations and $20.2 \%$ of the weights. Switching to a quantized PB-NOMS decoder with $q_{\mathrm{w}}=q_{\mathrm{ch}}=q_{\mathrm{m}}=3$ causes a performance loss of $0.65 \mathrm{~dB}$. As previously, NBP with $\boldsymbol{H}_{\text {std }}$ offers the lowest complexity, but is clearly not competitive.

\section{CONCLUSION}

We proposed a novel pruning-based neural belief propagation (PB-NBP) decoder for short linear block codes. The proposed decoder is based on pruning a large overcomplete parity-check matrix and uses different parity-check equations in each decoding iteration. For Reed-Muller codes and a polar code, we demonstrated a performance close to ML decoding. For a short, standardized LDPC code, we showed that we can outperform conventional $\mathrm{B}_{3} \mathrm{P}$ decoding at a reduced complexity. The proposed PB-NBP decoders outperform NBP with large overcomplete parity-check matrices while providing a lower complexity. In the case of a $(3,7)$ Reed-Muller code, the PBNBP decoder outperforms the NBP decoder by $0.28 \mathrm{~dB}$ at a BLER of $10^{-4}$ while only requiring $3 \%$ of the check nodes and $21 \%$ of the weights. Further, it performs within $0.27 \mathrm{~dB}$ from ML decoding. In all scenarios, our approach outperforms the original NBP and MBBP. We also applied the proposed pruning-based decoder to NOMS and introduced a quantized pruning-based neural offset min-sum (PB-NOMS) decoder which allows joint optimization of the weights, offsets, and quantization. With messages, weights, and offsets quantized with 5 bits, PB-NOMS achieves a performance $0.5 \mathrm{~dB}$ and $1.7 \mathrm{~dB}$ from ML for a Reed-Muller and LDPC code, respectively. A polar code with 3 bits quantization performs $1 \mathrm{~dB}$ away from ML. The proposed approach can readily be applied to other linear block codes such as $\mathrm{BCH}$ codes, with similar gains over the original NBP decoder expected. Furthermore, additional constraints can be introduced in the training process to allow for a more practical decoder. 


\section{APPENDIX \\ HYPERPARAMETERS}

The PB-NBP decoders are trained with a batch size of 128 in the case of the Reed-Muller code $\operatorname{RM}(2,5)$ and the LDPC code, and a batch size of 64 for the Reed-Muller code $\operatorname{RM}(3,7)$ and the polar code. As an optimizer, the Adam optimizer with a learning rate of 0.001 is employed. Initially, $\eta=1.0$ and every 3000 batches $\eta$ is decreased by multiplying it by 0.8 . After each pruning step, $\eta$ is reset to its initial value. The maximum number of batches per pruning step is $10^{5}$ for the $\operatorname{RM}(2,5)$ and the $\operatorname{LDPC}$ code, and $2 \cdot 10^{5}$ for the $\operatorname{RM}(3,7)$ and the polar code, but the next pruning step is performed earlier if the average loss over 100 batches stops decreasing.

The PB-NOMS decoders are trained starting from the pruned parity-check matrices. For the $\operatorname{RM}(2,5)$ and the LDPC code, we use $1.5 \cdot 10^{5}$ batches and for the $\operatorname{RM}(3,7)$ and the polar code $3 \cdot 10^{5}$ batches. As for the PB-NBP decoders, the learning rate is set to 0.001 and every 3000 batches $\eta$ is decreased by multiplying it by 0.8 .

\section{REFERENCES}

[1] E. Nachmani, Y. Be'ery, and D. Burshtein, "Learning to decode linear codes using deep learning," in Proc. Annu. Allerton Conf. Commun., Control, Comput., Allerton, IL, USA, Sep. 2016, pp. 341-346.

[2] E. Nachmani, E. Marciano, L. Lugosch, W. J. Gross, D. Burshtein, and Y. Be'ery, "Deep learning methods for improved decoding of linear codes," IEEE J. Sel. Topics Signal Process., vol. 12, no. 1, pp. 119-131, Feb. 2018.

[3] M. Lian, F. Carpi, C. Häger, and H. D. Pfister, "Learned beliefpropagation decoding with simple scaling and SNR adaptation," in Proc. IEEE Int. Symp. Inf. Theory (ISIT), Paris, France, Jul. 2019, pp. 161165.

[4] T. Gruber, S. Cammerer, J. Hoydis, and S. ten Brink, "On deep learningbased channel decoding," in Proc. Annu. Conf. Inf. Sci. Syst. (CISS), Baltimore, MD, USA, May 2017.

[5] L. Lugosch and W. J. Gross, "Neural offset min-sum decoding," in Proc. IEEE Int. Symp. Inf. Theory (ISIT), Aachen, Germany, Aug. 2017, pp. 1361-1365.

[6] T. R. Halford and K. M. Chugg, "Random redundant soft-in soft-out decoding of linear block codes," in Proc. IEEE Int. Symp. Inf. Theory (ISIT), Seattle, WA, USA, Jun. 2006, pp. 2230-2234.

[7] M. P. Fossorier, M. Mihaljevic, and H. Imai, "Reduced complexity iterative decoding of low-density parity check codes based on belief propagation," IEEE Trans. Commun., vol. 47, no. 5, pp. 673-680, May 1999.

[8] J. Chen and M. P. Fossorier, "Density evolution for two improved BPbased decoding algorithms of LDPC codes," IEEE Commun. Lett., vol. 6, no. 5, pp. 208-210, May 2002.
[9] M. Bossert and F. Hergert, "Hard- and soft-decision decoding beyond the half minimum distance - an algorithm for linear codes," IEEE Trans. Inf. Theory, vol. 32, no. 5, pp. 709-714, Sep. 1986.

[10] A. Kothiyal, O. Y. Takeshita, W. Jin, and M. Fossorier, "Iterative reliability-based decoding of linear block codes with adaptive belief propagation," IEEE Commun. Lett., vol. 9, no. 12, pp. 1067-1069, Dec. 2005.

[11] J. Jiang and K. R. Narayanan, "Iterative soft-input soft-output decoding of Reed-Solomon codes by adapting the parity-check matrix," IEEE Trans. Inf. Theory, vol. 52, no. 8, pp. 3746-3756, Aug. 2006.

[12] T. Hehn, J. Huber, O. Milenkovic, and S. Laendner, "Multiple-bases belief-propagation decoding of high-density cyclic codes," IEEE Trans. Commun., vol. 58, no. 1, pp. 1-8, Jan. 2010.

[13] E. Santi, C. Häger, and H. D. Pfister, "Decoding Reed-Muller codes using minimum-weight parity checks," in Proc. IEEE Int. Symp. Inf. Theory (ISIT), Vail, CO, USA, Jun. 2018, pp. 1296-1300.

[14] Y. Bengio, N. Léonard, and A. Courville, "Estimating or propagating gradients through stochastic neurons for conditional computation," Aug. 2013. [Online]. Available: http://arxiv.org/abs/1308.3432

[15] P. Yin, J. Lyu, S. Zhang, S. Osher, Y. Qi, and J. Xin, "Understanding straight-through estimator in training activation quantized neural nets," in Proc. Int. Conf. Learning Representations (ICLR), New Orleans, LA, USA, Mar. 2019.

[16] V. Nair and G. E. Hinton, "Rectified linear units improve restricted Boltzmann machines," in Proc. Int. Conf. Machine Learning (ICML), Haifa, Israel, Jun. 2010, pp. 807-814.

[17] D. P. Kingma and J. Ba, "Adam: A method for stochastic optimization," in Proc. Int. Conf. Learning Representations (ICLR), San Diego, CA, USA, May 2015, pp. 1-15.

[18] M. Abadi et al. (2015) TensorFlow: Large-scale machine learning on heterogeneous systems. [Online]. Available: https://www.tensorflow.org/

[19] B. Smith, M. Ardakani, W. Yu, and F. R. Kschischang, "Design of irregular LDPC codes with optimized performance-complexity tradeoff," IEEE Trans. Commun., vol. 58, no. 2, pp. 489-499, Feb. 2010.

[20] J. Frankle and M. Carbin, "The lottery ticket hypothesis: Finding sparse, trainable neural networks," in Proc. Int. Conf. Learning Representations (ICLR), New Orleans, LA, USA, Mar. 2019.

[21] A. Buchberger, C. Häger, H. Pfister, L. Schmalen, and A. Graell i Amat, "Source code," https://github.com/bbgra/pruning_nbp, 2020.

[22] J. Max, "Quantizing for minimum distortion," IRE Trans. Inf. Theory, vol. 6, no. 1, pp. 7-12, Mar. 1960.

[23] S. P. Lloyd, "Least squares quantization in PCM," vol. 28, no. 2, pp. 129-137, Mar. 1982.

[24] "Short block length LDPC codes for TC synchronization and channel codding (CCSDS 231.1-O-1)," Consultative Committee for Space Data Systems (CCSDS), Tech. Rep., Apr. 2015.

[25] M. Helmling, S. Scholl, F. Gensheimer, T. Dietz, K. Kraft, S. Ruzika, and N. Wehn, "Database of channel codes and ML simulation results," www.uni-kl.de/channel-codes, 2019.

[26] M. Bardet, V. Dragoi, A. Otmani, and J. P. Tillich, "Algebraic properties of polar codes from a new polynomial formalism," in Proc. IEEE Int. Symp. Inf. Theory (ISIT), Barcelona, Spain, Aug. 2016, pp. 230-234. 